\title{
Dynamic Hedge Fund Portfolio Construction
}

\author{
Richard D. F. Harris \\ University of Exeter \\ Murat Mazibas \\ University of Exeter \\ Paper No 09/07 \\ November 2009
}

\begin{abstract}
In this paper, we provide further evidence on the use of multivariate conditional volatility models in hedge fund risk measurement and portfolio allocation, using monthly hedge fund index return data for the period 1990 to 2009. Building on Giamouridis and Vrontos (2007), we consider a broad set of multivariate GARCH models as well as the simpler exponentially weighted moving average (EWMA) estimator of RiskMetrics (1996). We find that while multivariate GARCH models provide some improvement in portfolio performance over static models, they are generally dominated by the EWMA model. In particular, in addition to providing better risk-adjusted performance, the EWMA model leads to dynamic allocation strategies that have substantially lower turnover and could therefore be expected to involve lower transaction costs. Moreover, we show that these results are robust across low-volatility and high-volatility sub-periods.
\end{abstract}

Keywords: Hedge fund returns; Funds of funds; Multivariate conditional volatility; Portfolio optimisation. 


\section{Introduction}

The hedge fund industry has grown rapidly over the last two decades, from as few as 300 funds in 1990 to more than 9,000 today. These funds currently manage assets of more than 2.5 trillion USD (Ineichen and Silberstein, 2008). Hedge fund managers typically employ dynamic trading strategies with frequent re-balancing, and often make extensive use of derivatives, short positions and leverage. Primarily aimed at institutional investors and high net worth individuals, hedge funds have recently become more widely accessible through the emergence of 'funds of funds', which hold portfolios of hedge fund investments that are sold to a wider investor base. These funds provide a broad exposure to the hedge fund sector and diversify the risks associated with an investment in individual funds. Since their inception, these funds have become extremely popular. Indeed, by 2006, there were over two thousand funds of hedge funds listed in the Hedge Fund Research (HFR) database.

A number of studies have examined portfolio optimisation in a hedge fund context. However, the optimal portfolio allocation across individual hedge funds is complicated by the fact that owing to the strategies that hedge fund managers typically adopt, hedge fund returns are far from normally distributed, usually exhibiting very significant negative skewness and excess kurtosis (see, for example, Amin and Kat, 2001; Lo, 2001; Brooks and Kat, 2002; Fung and Hsieh, 1997a, 2001; Agarwal and Naik, 2001). Portfolio optimisation in the presence of such nonnormality in hedge fund returns generally leads to very different portfolio allocations than those implied by mean-variance analysis (see, for example, McFall and Lamm, 2003; Fung and Hsieh, 1997b; Cvitanic et al., 2003; Terhaar et al., 2003; Popova et al,. 2003).

While the non-normality of hedge fund returns is by now well-established, much less attention has been paid to the dynamic properties of hedge fund risk. In particular, the literature on hedge fund portfolio optimisation has typically assumed a constant covariance structure of hedge fund returns. The assumption of a time-invariant variance-covariance matrix of hedge fund returns potentially leads to inaccuracies in the measurement of hedge fund risk and the optimisation of hedge fund portfolios, particularly over shorter horizons where time-variation in the covariance matrix of 
returns is most pronounced. Indeed, Giamouridis and Vrontos (2007) show that the use of multivariate conditional volatility models improves the optimisation of hedge fund portfolios, and provides a more accurate tool for tail-risk measurement. They employ two static models (the sample covariance matrix and an implicit factor model) and three dynamic models (two implicit factor GARCH models and a regime switching dynamic correlations model), and compare the out-of-sample performance of optimised monthly and quarterly rebalanced portfolios of the HFR indices for the period January 2002 to August 2005. In the mean-variance framework, the use of dynamic models generates portfolios of hedge fund indices with lower out-of-sample risk and higher realized returns. Using a mean-CVaR framework, which implicitly accommodates the non-normality of hedge fund returns, they show that dynamic optimisation models are also more successful in reducing left tail risk.

In this paper, we extend the results of Giamouridis and Vrontos (2007) to provide further evidence on the usefulness of multivariate conditional volatility models in hedge fund portfolio optimisation using monthly index return data from the Hedge Fund Research (HFR) database for the period 1990 to 2009. In particular, we consider a broader set of volatility models, including a number of additional multivariate GARCH models and the much simpler exponentially weighted moving average (EWMA) estimator of RiskMetrics (1996). We find that while multivariate GARCH models provide some improvement in portfolio performance over static models, they are generally dominated by the EWMA model. In particular, in addition to providing better risk-adjusted performance, the EWMA model leads to dynamic allocation strategies that have substantially lower turnover and could therefore be expected to involve lower transaction costs. To test the robustness of our results, we apply the models to two sub-periods, one representing a bull market (2002 to 2005) and one representing a bear market (2006 to 2009). The EWMA model appears to work well in both favourable and unfavourable market conditions.

The outline of the paper is as follows. In the following section, we describe the data used in the empirical analysis, the multivariate volatility models and the evaluation methodology. Section 3 reports the empirical results. Section 4 provides a summary and some concluding remarks. 


\section{Data and Methodology}

\subsection{Data}

We use monthly data on hedge fund index returns obtained from Hedge Fund Research (HFR). In line with Amenc and Martellini (2002), McFall and Lamm (2003), Agarwal and Naik (2004), Morton et al. (2006) and Giamouridis and Vrontos (2007), the indices are classified into the following investment strategies: equity hedge, macro, relative value arbitrage, event driven, convertible arbitrage, distressed securities, equity market neutral and mergers arbitrage. We use data for the period January 1990 to September 2009 (237 observations). This period covers a number of crises (e.g. the Mexican crisis, the Asian financial crisis, the default of the Russian government on its debt, the collapse of Long Term Capital Management, the collapse of the dotcom bubble and the most recent credit crisis). The initial estimation period covers the period January 1990 to December 2001 (144 observations). The out-ofsample forecast period is divided into two sub-periods: January 2002 to August 2005, representing relatively favourable market conditions (44 observations) and September 2005 to September 2009, representing more extreme market conditions (49 observations). Summary statistics for the hedge fund return series are reported in Table 1.

[Table 1]

Panel A reports various statistics for the different hedge fund investment strategies for the full sample of 237 observations. The characteristics of hedge fund returns are very heterogeneous. Some strategies (such as equity hedge and event driven) have relatively higher average returns and volatility. These are often thought of as return enhancers, used to substitute some fraction of the equity holdings in an investor's portfolio (see Amenc and Martellini, 2002). Other strategies (such as relative value arbitrage and equity market neutral) have lower average return and volatility, and can be regarded as a substitute for some fraction of the fixed income or cash holdings in an investor's portfolio. All strategies except macro display negative skewness, and all are leptokurtic, particularly relative value arbitrage, convertible arbitrage and mergers arbitrage. The null hypothesis of normality is strongly rejected in all cases. Panel B 
reports the basic time series properties of hedge fund returns. In particular, it reports the first five autocorrelation coefficients, the Ljung-Box portmanteau test for serial correlation up to $10 \mathrm{lags}$, the ARCH test of Engle (1982) and the DCC test of Engle and Sheppard (2001). All the hedge fund indices display highly significant positive autocorrelations and the ARCH test suggests that there is evidence of significant volatility clustering for all strategies except macro and mergers arbitrage. The DCC test, which tests the null hypothesis of constant correlation is tested against the alternative of dynamic conditional correlation, suggests that the data exhibit timevarying conditional correlations and hence motivates the use of dynamic conditional covariance models. Panel $\mathrm{C}$ reports the pair-wise correlations computed for the single strategies. Hedge fund returns exhibit correlations from 0.23 (between macro and convertible arbitrage) to 0.84 (between event driven and distressed securities). Generally, correlations between the different strategies is relatively moderate, which is a desirable property in the construction of funds of hedge funds.

\subsection{Methodology}

We define the first and second moments of $N$ hedge fund returns, conditional on the information set $\Omega$, as follows

$$
\begin{aligned}
& R_{t}=\mu+\varepsilon_{t} \\
& \varepsilon_{t} \mid \Omega_{t-1} \sim D\left(0, H_{t}\right)
\end{aligned}
$$

where $R_{t}$ is the $N \times 1$ vector of hedge fund returns in period $t$ with elements $R_{i, t}$, $i=1, \ldots, N, \mu$ is the $N \times 1$ vector of mean returns with elements $\mu_{i}, i=1, \ldots, N$ and $H_{t}$ is the $N \mathrm{x} N$ covariance matrix with diagonal elements $\sigma_{i, t}^{2}$ and off-diagonal elements $\sigma_{i j, t}$, $i, j=1, \ldots, N . D($.) is any location-scale family distribution. We consider an investor in the $N$ hedge funds who wishes to minimise the variance of portfolio returns in each period $t$ subject to a minimum return constraint and short selling constraints. The portfolio optimisation problem can therefore be written as

$$
\min _{w_{t}} \operatorname{var}\left(R_{p, t}\left(w_{t}\right)\right)
$$


where $R_{p, t}$ is the return of the hedge fund portfolio on day $t, \operatorname{var}\left(R_{p, t}\right)=w_{t}{ }_{t} H_{t} w_{t}$ is the conditional portfolio variance, $w_{t}$ is the $N \times 1$ vector of portfolio weights, $\mu_{p, t}$ is the portfolio expected return and $R_{0}$ is the target portfolio return. In modelling the covariance matrix of hedge fund returns, $H_{t}$, we employ two static models and six dynamic models. Each of these models is described below.

\section{Sample Covariance Model}

The simplest static model of the variance-covariance matrix of hedge fund returns is the sample covariance matrix of historical returns, given by

$$
H^{S C}=\frac{1}{T-1} \sum_{t=1}^{T}\left(R_{t}-\bar{R}\right)\left(R_{t}-\bar{R}\right)^{\prime}
$$

where $\bar{R}=(1 / T) \sum_{t=1}^{T} R_{t}$ is the $N \mathrm{x} 1$ vector of sample mean returns. The sample covariance model is perhaps the most commonly used estimator of the return covariance matrix (Amenc \& Martellini, 2002).

\section{Implicit Factor Model}

The implicit factor model utilised by Fung and Hsieh (1997a), Amenc and Martellini (2002), Alexander and Dimitriu (2004) and Giamouridis and Vrontos (2007), assumes that returns are generated by a multifactor model. Under the implicit factor model, the covariance matrix of hedge fund returns is given by

$$
H^{I F}=\Lambda \Sigma^{I F} \Lambda^{\prime}+V
$$


where $\Lambda$ is the $N \times K$ matrix of factor loadings, $\Sigma^{I F}$ is the $K \times K$ diagonal factor covariance matrix, $V$ is a diagonal matrix with elements in the main diagonal $\sigma_{\varepsilon_{i}}^{2}=\operatorname{var}\left(u_{i, t}\right)$ and $u_{i, t}$ is the idiosyncratic return with respect to the $K$ factors.

Regime Switching Dynamic Correlation (RSDC) Model

Following Giamouridis and Vrontos (2007), we employ the RSDC model of Pelletier (2006). We estimate the model using the two-step procedure described by Engle (2002). In the first step, we estimate a univariate volatility model for each hedge fund index, and in the second step, we estimate the parameters of the correlation matrix, conditional on the volatility estimates. We assume that there are two regimes and employ the GARCH $(1,1)$ specification in modelling the conditional variances. Under the RSDC model, the variance-covariance matrix of hedge fund returns is given as

$$
H_{t}^{R S D C}=D_{t} P_{t} D_{t}
$$

where $D_{t}=\operatorname{diag}\left(h_{11, t}^{-1 / 2} \ldots h_{N N, t}^{-1 / 2}\right)$ is the $N \mathrm{x} N$ diagonal matrix of the inverse standard deviations of hedge fund returns and $P_{t}$ is the $N \times N$ regime switching correlation matrix. The RSDC model stands somewhere between the Constant Conditional Correlation (CCC) model of Bollerslev (1990), in which conditional correlations are time-invariant, and the Dynamic Conditional Correlation (DCC) model of Engle (2002), in which conditional correlations are time-varying. Further details of the RSDC model can be found in Pelletier (2006).

\section{Orthogonal GARCH Model}

The Orthogonal GARCH model of Alexander (2001) is a generalization of the factor GARCH model introduced by Engle et al. (1990). For $m$ factors the Orthogonal GARCH model is defined as

$$
\varepsilon_{t}=V^{1 / 2} u_{t}=\Lambda_{m} f_{t}
$$


where $V=\operatorname{diag}\left(s_{1}^{2}, \ldots, s_{N}^{2}\right)$ is a diagonal matrix comprising the variance of $\varepsilon_{i t}$, $\Lambda_{m}=P_{m} \operatorname{diag}\left(l_{1}^{1 / 2} \ldots l_{m}^{1 / 2}\right)$ is the $(N \mathrm{x} m)$ matrix of factor loadings, $P_{m}$ is the matrix of mutually orthogonal eigenvectors and $l_{1} \geq \ldots \geq l_{m}>0$ are the $m$ largest eigenvalues of the empirical correlation matrix of $u_{t}$. As $f_{t}$ is defined as the random process with $E_{t-1}\left(f_{t}\right)=0$ and $\operatorname{var}_{t-1}\left(f_{t}\right)=\Sigma_{t}=\operatorname{diag}\left(\sigma_{f_{1 t}}^{2}, \ldots, \sigma_{f_{m t}}^{2}\right), \quad$ the conditional variancecovariance matrix can be defined as

$$
H_{t}^{O R T H}=V^{1 / 2} V_{t} V^{1 / 2}
$$

where $V_{t}=\Lambda_{m} \Sigma_{t} \Lambda_{m}^{\prime}$. Further details of Orthogonal GARCH model can be found in Alexander (2001) and Bauwens et al (2006).

\section{Dynamic Conditional Correlation (DCC) Model}

The DCC model of Engle and Sheppard (2001) and Engle (2002) is defined as

$$
H_{t}^{D C C}=D_{t} P_{t} D_{t}
$$

where $D_{t}=\operatorname{diag}\left(h_{11, t}^{-1 / 2} \ldots h_{N N, t}^{-1 / 2}\right)$ is the $N \mathrm{x} N$ diagonal matrix of the inverse standard deviations of returns and $P_{t}$ is the $N \mathrm{x} N$ dynamic correlation matrix. There are also other versions of the DCC model proposed by Tse and Tsui (2002) and Christodoulakis and Satchell (2002).

\section{Flexible Multivariate GARCH Model}

The Flexible Multivariate GARCH model proposed by Ledoit et al. (2003) estimates the conditional covariance matrices within the framework of the diagonal vech GARCH model. The diagonal vech model is given by

$$
H_{t}^{V E C}=C+A v e c h\left(H_{t-1}^{V E C}\right)+B v e c h\left(\varepsilon_{t} \varepsilon_{t}^{\prime}\right)
$$


where $C$ is an $N(N+1) / 2 \times 1$ vector and $A$ and $B$ are $N(N+1) / 2 \times N(N+1) / 2$ matrices. The diagonal vech model has a number of well-documented shortcomings. Firstly, the number of parameters grows at the rate $N^{2}$, so for anything more than just a few assets, parameter estimation is infeasible. Secondly, the conditional covariance matrix is not guaranteed to be positive semi-definite. However, Ledoit et al. (2003) propose a two-step estimation method that ensures positive semi-definiteness. In the first step, a bivariate diagonal vech model is estimated for each pair of assets and the estimated parameters are stacked into matrices $\hat{C}, \hat{A}$ and $\hat{B}$. In the second step, $\hat{C}, \hat{A}$ and $\hat{B}$ are transformed in such a way that ensures that the resulting covariance matrix, $H_{t}^{F L E X}$, is positive semi-definite.

\section{BEKK Model}

The BEKK model of Engle and Kroner (1995) generalises the univariate GARCH model to the multivariate case. The $\operatorname{BEKK}(1,1, N)$ specification is given by

$$
H_{t}^{B E K K}=C C^{\prime}+\sum_{n=1}^{N} A^{\prime} H_{t-1} A+\sum_{n=1}^{N} B^{\prime} \varepsilon_{t-1} \varepsilon_{t-1}^{\prime} B
$$

where $A, B$ and $C$ are $N \mathrm{x} N$ parameter matrices and $C$ is lower triangular. This model is also equivalent to a Factor $\operatorname{GARCH}(1,1, N)$ model under certain conditions (see Bauwens et al., 2006).

\section{Exponentially Weighted Moving Average (EWMA) Model}

The EWMA model, popularised by its use in the estimation of Value at Risk by JP Morgan in their RiskMetrics software, is a special case of the diagonal vech GARCH $(1,1)$ model. Under the EWMA model, the variance-covariance matrix of returns is given by

$$
H_{t}^{E W M A}=\lambda R_{t-1} R_{t-1}^{\prime}+(1-\lambda) H_{t-1}
$$


where $\lambda$ is a decay factor, commonly set to a value of 0.94 for daily data. The mean return is assumed to be zero, as is common in practice.

We initially estimate each of the six conditional volatility models using the first 144 observations to generate a one-month ahead out-of-sample forecast of the conditional covariance matrix for month 145. Where appropriate, starting parameter values for this initial estimation were chosen on the basis of a grid-search procedure to maximize the likelihood function. The estimation sample was then rolled forward by one month, the models re-estimated and used to generate out-of-sample forecasts for month 146, and so on until the end of the sample. At each iteration, the starting parameter values for each model were set to the values estimated in the previous iteration. The models were estimated by Quasi Maximum Likelihood function with a Gaussian conditional distribution, using the $\mathrm{BHHH}$ algorithm.

\subsection{Evaluation}

Each month, we use the forecast conditional covariance matrix to estimate the weights of two optimal portfolios. The first is a 'conservative' portfolio, which does not have a target expected return, i.e. the minimum-variance portfolio. The second is an 'aggressive' portfolio with an annualised target expected return of either 15.5 percent or 13.5 percent, depending on the period analysed. In all cases, in order to force diversification, we constrain the maximum weight in any one index to be 70 percent. We report results for a holding period of one month. The results are qualitatively similar using longer holding periods of three and six months. Following Agarwal and Naik (2004) and Giamouridis and Vrontos (2007), for each portfolio and each holding period, we estimate a number of evaluation criteria, which are defined below.

Return

$$
R_{p, t+1}=w_{t} R_{t+1}
$$


Standard deviation

$$
\sigma_{p, t+1}=w_{t}^{\prime} H_{t+1} w_{t}
$$

Conditional Sharpe ratio

$$
\operatorname{CSR}_{P, t+1}=\frac{R_{P, t+1}}{\sigma_{P, t+1}}
$$

Turnover

$$
T_{t+1}=\sum_{i=1}^{N}\left|w_{i, t+1}-w_{i, t}\right|
$$

This measures the fraction of the portfolio that must be liquidated and reinvested each month.

\section{Conditional value at risk}

$$
C V a R_{t+1}^{\alpha}=\frac{\int_{-\infty}^{-V a R_{t+1}^{\alpha}} z f_{R_{t}}(z) d z}{F_{R_{t}}\left(-V a R_{t+1}^{\alpha}\right)}
$$

where $\alpha$ is the CVaR confidence level, $f_{R_{t}}$ is the probability density function, $F_{R_{t}}$ is the cumulative distribution function and $\operatorname{VaR}_{t+1}^{\alpha}=-F_{R_{t}}^{-1}(1-\alpha)$. We estimate $\mathrm{CVaR}$ at the 90 percent, 95 percent and 99 percent confidence levels.

\section{Empirical Results}

We test the out-of-sample forecast performance of constant and dynamic conditional covariance models in an investment exercise for the out-of-sample period January 2002 to September 2009. We divide the out-of-sample test period into two subperiods. The first, which is from January 2002 to August 2005, is the same out-of- 
sample period analyzed in Giamouridis and Vrontos (2007), and is a relatively favourable period for hedge funds. The second, from September 2005 to September 2009 , includes the recent financial crisis, and is hence a relatively volatile period for hedge funds. We provide empirical evidence for a conservative (i.e. minimum variance) portfolio and an aggressive (i.e. target return) portfolio. In the first period, to be comparable to Giamouridis and Vrontos (2007), we assume a target return for the aggressive portfolio of 15.5 percent. For the second period, when hedge fund returns were generally lower, we assume a target return of 13.5 percent. By considering these two periods, we are able to assess the out-of-sample performance of the various conditional covariance models under both normal and extreme market conditions. For each of the eight models, we report both the mean and median values of the realized return, portfolio standard deviation, conditional Sharpe ratio, portfolio turnover and CVaR tail-risk measure. The statistical significance of pair-wise differences in these measures between the eight models were tested using a t-test (for differences in mean values) and the Wilcoxon test (for differences in median values).

\section{Period 1: Normal Market Conditions}

Table 2 reports the mean and median values of the risk-return metrics for the estimated portfolios for Period 1. Realized returns are uniformly higher for the aggressive portfolio than for the conservative portfolio, but portfolio standard deviation is also uniformly higher. The Sharpe ratios are similar for the two portfolios. The static models (SC and IF) perform poorly in terms of risk-adjusted return for both portfolios, characterised by notably lower returns for the conservative portfolio and notably higher risk for the aggressive portfolio. As expected, however, they have the lowest turnover of almost all of the models, reflecting their static nature. The RSDC model offers a substantial improvement over the static models for both portfolios, offering higher returns for the conservative portfolio and lower risk for the aggressive

portfolio, but has much higher turnover. In terms of risk-adjusted performance, the RSDC model generates a Sharpe ratio of 0.74 for the conservative portfolio and 0.75 for the aggressive portfolio, compared with 0.49 and 0.51 , respectively for the SC model and 0.49 and 0.50 , respectively, for the IF model. The superior performance of the RSDC model relative to the static model is statistically significant. These findings 
are consistent with Giamouridis and Vrontos (2007) who report results for the same portfolios optimised with the SC, IF and RSDC models over the same period.

Of the remaining GARCH models, the DCC and FLEX models provide performance that is very similar to that of the RSDC model. In contrast, however, the ORTH and BEKK models perform notably worse, both generating significantly lower returns for the conservative portfolio and significantly higher risk for the aggressive portfolio. The best performing GARCH model in risk-adjusted return terms is the FLEX model for the conservative portfolio (with a Sharpe ratio of 0.78 ) and the RSDC model for the aggressive portfolio (with a Sharpe ratio of 0.75 ). However, for the conservative portfolio, the much simpler EWMA model generates the highest Sharpe ratio of 0.87. This improvement in risk-adjusted performance comes primarily from a reduction in risk. For the aggressive portfolio, the EWMA model offers risk-adjusted performance that is very similar to that of the RSDC, DCC and FLEX models. A notable feature of the EWMA model, however, is that it generates very low turnover for both the conservative and aggressive portfolios, and indeed, has similar turnover to that of the static models. The EWMA model could be expected, therefore, to generate lower transaction costs relative to the GARCH models that we consider. The $\mathrm{CVaR}$ estimates re-enforce the conclusions about risk reached above. In particular, the EWMA model generates a conservative portfolio that is substantially less riskier than any of the GARCH models, or indeed the two static models. For the aggressive portfolio, there is little difference in the CVaR among the best performing GARCH models.

\section{Period 2: Extreme Market Conditions}

Table 3 reports the mean and median values of the risk-return metrics for the estimated portfolios for Period 2. Realized returns are generally higher for the aggressive portfolio than for the conservative portfolio, although there are some exceptions (most notably the RSDC model), and portfolio standard deviation is significantly higher. Owing to the higher volatility of this period, risk-adjusted returns, as measured by the Sharpe ratio, are uniformly higher for the conservative portfolio than for the aggressive portfolio. In contrast with Period 1, the performance of the static models (SC and IF) in Period 2 is similar to that of the dynamic models in 
terms of realised return and standard deviation. In terms of risk-adjusted returns, the static models have lower mean Sharpe ratios, but the median values are similar to those of the other models, suggesting perhaps that the mean values in this case are driven by outliers. Similar to Period 1, the static models have much lower turnover owing to their relatively unresponsive nature. Among the dynamic models, the RSDC and EWMA models provide the best risk-adjusted return performance for the conservative portfolio and the RSDC, EWMA and DCC models provide the best performance for the aggressive portfolio.

Comparing the results for Period 1 and Period 2, we see that the deterioration in performance is greater for the aggressive portfolio than for the static portfolio, which is to be expected given the unfavourable conditions prevailing in Period 2. In particular, the standard deviation of returns is generally higher in Period 2 for the aggressive portfolio, while the level of returns is substantially lower. For the conservative portfolio, the standard deviation of returns is also generally higher in Period 2 than in Period 1, but the median level of returns is in many cases higher. The EWMA model is again notable for having the lowest turnover of the dynamic models, and for the aggressive portfolio, it is similar to that of the two static models. In terms of tail risk, the aggressive portfolios have significantly higher CVaR than the conservative portfolios, but there is not a systematic difference between the dynamic models and the static models. Of the dynamic models, the EWMA model and the FLEX model offer the lowest tail risk at all three CVaR levels for both the conservative and aggressive portfolios. A number of the dynamic models have substantially higher tail risk than the static models. This is especially true of the RSDC and ORTH and DCC models.

\section{Conclusion}

In this paper, we build on the analysis of Giamouridis and Vrontos (2007) and provide further evidence on the performance of dynamic conditional covariance models for the optimisation of funds of hedge funds. We do so by considering a much broader set of conditional covariance models, and a longer out-of-sample evaluation period. In particular, we consider not only additional GARCH models, but also the much simpler RiskMetrics EWMA model. Moreover, we separately consider evaluation 
periods of favourable and unfavourable conditions for the hedge fund industry. In the first out-of-sample period, which is a relatively favourable period, we confirm the findings of Giamouridis and Vrontos (2007) and show that GARCH models are able to provide a substantial improvement in terms of risk-return trade-off over the static models, for the optimisation of both conservative and aggressive portfolios. However, in most cases these improvements come at the expense of higher portfolio turnover and rebalancing expenses. In contrast, the EWMA model, which is the most parsimonious of the dynamic models, offers a superior risk-return trade-off and, moreover, does so with rebalancing costs that are no higher than those of the static models. In the second out-of-sample period, which is characterised by much greater volatility and generally unfavourable conditions for the hedge fund industry, dynamic models again tend to outperform static models, providing a superior risk-return tradeoff, although the differences are less marked than during the favourable conditions of the first out-of-sample period. The best performing models in terms of risk-adjusted returns are the RSDC, EWMA and DCC models, but of these, the EWMA model is again notable for generating portfolios that have substantially lower turnover and less tail risk. Our findings therefore confirm the advantages of dynamic models over static models, but favour the use of simple models over more sophisticated models. 


\section{References}

Agarwal, V., N.Y. Naik (2001). 'Risks and Portfolio Decisions Involving Hedge Funds. Technical Report', London Business School.

Agarwal, V., N.Y. Naik (2004). 'Risks and Portfolio Decisions Involving Hedge Funds', Review of Financial Studies 17 (1), 63-98.

Alexander, C., (2001). 'Orthogonal GARCH', In: Alexander, C. (Ed.), Mastering Risk, Financial Times, vol. 2. Prentice Hall, pp. 21-38.

Alexander, C., A. Dimitriu (2004). 'The Art Of Investing in Hedge Funds: Fund Selection and Optimal Allocation', ICMA Centre Discussion Papers in Finance, University of Reading.

Amenc, N., Martellini, L., (2002). 'Portfolio Optimization and Hedge Fund Style Allocation Decisions', Journal of Alternative Investments 5, 7-20.

Amin, G.S., H.M. Kat (2001). 'Hedge fund performance 1990-2000. Do the "Money Machines” Really Add Value?', Working Paper, University of Reading.

Bauwens, L., S. Laurent, J. V. K. Rombouts (2006). 'Multivariate GARCH Models: A survey', Journal of Applied Econometrics, 21, 79-109.

Bernardo, A.E., Ledoit, O. (2000). 'Gain, Loss, and Asset Pricing'. Journal of Political Economy, 108 (1).

Brooks, C., Kat, H.M., (2002). 'The statistical Properties of Hedge Fund Index Returns and Their Implications for Investors', The Journal of Alternative Investments, 5, 26-44.

Cvitanic', J., A. Lazrak, L. Martellini, F. Zapatero (2003). 'Optimal Allocation to Hedge Funds: An Empirical Analysis', Quantitative Finance 3, 1-12.

Christodoulakis G.A., S.E. Satchell (2002). 'Correlated ARCH: Modelling The TimeVarying Correlation Between Financial Asset Returns', European Journal of Operations Research 139: 351-370.

Eling, M. (2008). 'Does Hedge Fund Performance Persist? Overview and New Empirical Evidence', Working Paper, University of St.Gallen, available at SSRN.

Engle, R. F. (1982). 'Autoregressive Conditional Heteroscedasticity with Estimates of Variance of United Kingdom Inflation', Econometrica 50:987-1008.

Engle, R. F. (2002). 'Dynamic Conditional Correlation: A Simple Class of Multivariate Generalized Autoregressive Conditional Heteroskedasticity Models', Journal of Business and Economic Statistics, 20, 339-350.

Engle, R., T. Ito, and W.-L. Lin (1990). 'Meteor Showers or Heat Waves? Heteroskedastic Intra-daily Volatility in the Foreign Exchange Market', Econometrica, 58, 525-542.

Engle, R., F. Kroner (1995). 'Multivariate Simultaneous Generalized ARCH', Econometric Theory, 11, 122-150.

Engle, R.F., K. Sheppard (2001). 'Theoretical and Empirical Properties of Dynamic Conditional Correlation Multivariate GARCH', NBER Working Paper No. W8554. Available at SSRN: 
Fung, W., D.A. Hsieh, (1997a). 'Empirical Characteristics of Dynamic Trading Strategies: The Case of Hedge Funds', Review of Financial Studies 10, 275-302.

Fung, W., D.A. Hsieh, (1997b). 'Is Mean-Variance Analysis Applicable to Hedge Funds?', Working Paper, Fuqua School of Business, Duke University.

Fung, W., D.A. Hsieh (2001). 'The Risk in Hedge Fund Strategies: Theory and Evidence from Trend Followers', The Review of Financial Studies 14, 313-341.

Giamouridis, D., I.D. Vrontos (2007). 'Hedge Fund Portfolio Construction: A Comparison of Static and Dynamic Approaches', Journal of Banking and Finance 31, 199-217.

Goodman, M., K. Shewer, R. Horwitz (2002). 'Integrating Market Correlation into Risk-Adjusted Return', Risk, June.

Ineichen, A., K. Silberstein (2008). 'AIMA's Roadmap to Hedge Funds', Alternative Investment Management Association, London.

Johnson, D., N. Macleod, C. Thomas (2002). 'A Framework for the Interpretation of Excess Downside Deviation', AIMA Newsletter, September, 14-16.

Ledoit O., P. Santa-Clara, M. Wolf (2003). 'Flexible Multivariate GARCH Modelling with an Application to International Stock Markets', The Review of Economics and Statistics 85: 735-747.

Lo, A.W. (2001). 'Risk Management for Hedge Funds: Introduction and Overview'. NBER Working Paper. Available at SSRN.

Markowitz, H.M. (1952). 'Portfolio Selection', Journal of Finance 7, 77-91.

McFall Lamm, R. (2003). 'Asymmetric Returns and Optimal Hedge Fund Portfolios', Journal of Alternative Investments 6 (2), 9-21.

Mitchell M., M., T. Pulvino (2001). 'Characteristics of Risk and Return in Risk Arbitrage', the Journal of Finance, 56 (6), 2135-2175.

Morton, D.P., E. Popova, I. Popova, (2006). 'Efficient Fund of Hedge Funds Construction under Downside Risk Measures', Journal of Banking and Finance 30 (2), 503-518.

Pelletier (2006). 'Regime Switching for Dynamic Correlations', Journal of Econometrics, 131(1-2), 445-473.

Popova, I., D.P. Morton, E. Popova (2003). 'Optimal Hedge Fund Allocation with Asymmetric Preferences and Distributions', Working Paper, University of Texas at Austin.

RiskMetrics (1996). Technical Document, 4th Edition, RiskMetrics.

Schneeweis, T., H.B. Kazemi, G.A. Martin (2002). 'Understanding Hedge Fund Performance: Research Issues Revisited-Part I', The Journal of Alternative Investments, 5 (3), 6-22.

Schneeweis, T., H.B. Kazemi, G.A. Martin (2003). 'Understanding Hedge Fund Performance: Research Issues Revisited-Part II', The Journal of Alternative Investments, 5 (4), 8-30. 
Silvennoinen, A., Terasvirta, T. (2008). 'Multivariate GARCH models'. To appear in T. G. Andersen, R. A. Davis, J.-P. Kreiss and T. Mikosch, eds. Handbook of Financial Time Series. New York: Springer.

Sortino, F.A., R.van der Meer (1991). 'Downside Risk'. The Journal of Portfolio Management, 17(4), 27-31.

Stutzer, M. J (2000). 'A Portfolio Performance Index', Financial Analysts Journal, $56(3), 52-61$.

Terhaar, K., R. Staub, B. Singer, (2003). 'Appropriate Policy Allocation for Alternative Investments', Journal of Portfolio Management 29 (3), 101-111.

Tse, Y. K., Tsui K. C. (2002). 'A Multivariate Generalized Autoregressive Conditional Heteroscedasticity Model with Time-Varying Correlations', Journal of Business and Economic Statistics, 20, 351-362. 
Table 1: Summary Statistics and Time Series Properties of Hedge Fund Return Series Panel A: Summary Statistics

\begin{tabular}{|c|c|c|c|c|c|c|c|c|}
\hline & Mean & Median & SD & Skew & Kurt. & Min. & Max. & Jarque-Bera \\
\hline Equity hedge & 1.15 & 1.31 & 2.68 & -0.24 & 4.91 & -9.46 & 10.88 & $38.23[0.00]$ \\
\hline Macro & 1.13 & 0.83 & 2.25 & 0.44 & 3.81 & -6.40 & 7.88 & $14.03[0.00]$ \\
\hline Relative value arbitrage & 0.85 & 0.91 & 1.31 & -2.23 & 16.97 & -8.03 & 5.72 & $2122.21[0.00]$ \\
\hline Event driven & 1.00 & 1.29 & 2.02 & -1.36 & 7.20 & -8.90 & 5.13 & $246.92[0.00]$ \\
\hline Convertible arbitrage & 0.74 & 1.00 & 1.97 & -3.24 & 32.50 & -16.01 & 9.74 & $9006.08[0.00]$ \\
\hline Distressed securities & 1.02 & 1.12 & 1.94 & -1.01 & 7.89 & -8.50 & 7.06 & $276.58[0.00]$ \\
\hline Equity market neutral & 0.63 & 0.58 & 0.94 & -0.17 & 4.19 & -2.87 & 3.59 & $15.16[0.00]$ \\
\hline Mergers arbitrage & 0.76 & 0.96 & 1.23 & -2.21 & 11.68 & -6.46 & 3.12 & $936.70[0.00]$ \\
\hline
\end{tabular}

Panel B: Basic Time Series Properties

\begin{tabular}{|c|c|c|c|c|c|c|c|c|}
\hline & $\operatorname{ACF}(1)$ & $\operatorname{ACF}(2)$ & $\operatorname{ACF}(3)$ & $\mathrm{ACF}(4)$ & $\operatorname{ACF}(5)$ & LB-Q(10) & $\operatorname{ARCH}(4)$ & $\begin{array}{l}\text { DCC test } \\
59.48[0.00]\end{array}$ \\
\hline Equity hedge & $0.26^{* * *}$ & $0.16^{* * *}$ & $0.09 * * *$ & $0.05 * * *$ & $-0.05 * * *$ & $29.53 * * *$ & $22.90 * * *$ & \\
\hline Macro & $0.16^{* *}$ & $-0.00 * *$ & 0.01 & $0.11 *$ & $0.17 * * *$ & $22.03 * *$ & 6.78 & \\
\hline Relative value arbitrage & $0.46 * * *$ & $0.27 * * *$ & $0.12 * * *$ & $0.11 * * *$ & $0.03 * * *$ & $74.43 * * *$ & $44.74 * * *$ & \\
\hline Event driven & $0.39 * * *$ & $0.17 * * *$ & $0.10 * * *$ & $0.06 * * *$ & $0.03 * * *$ & $48.13^{* * *}$ & $15.07 * * *$ & \\
\hline Convertible arbitrage & $0.61 * * *$ & $0.32 * * *$ & $0.16^{* * *}$ & $0.12^{* * *}$ & $-0.03 * * *$ & $134.58 * * *$ & $55.29 * * *$ & \\
\hline Distressed securities & $0.57 * * *$ & $0.28 * * *$ & $0.15 * * *$ & $0.14 * * *$ & $0.02 * * *$ & $108.66^{* * *}$ & $46.28 * * *$ & \\
\hline Equity market neutral & $0.16 * * *$ & $0.18 * * *$ & $0.20 * * *$ & $0.19 * * *$ & $0.08 * * *$ & $72.98 * * *$ & $16.81 * * *$ & \\
\hline Mergers arbitrage & $0.23 * * *$ & $0.08 * * *$ & $0.11 * * *$ & $0.00 * * *$ & $0.07 * * *$ & $26.92 * * *$ & 0.57 & \\
\hline \multicolumn{9}{|c|}{ Panel C: Correlation Structure of Individual Hedge Fund Strategies } \\
\hline & [ 1 ] & [ 2 ] & [3] & [ 4 ] & [ 5 ] & [ 6] & {$[7]$} & {$[8]$} \\
\hline Equity hedge & 1.00 & & & & & & & \\
\hline Macro & 0.56 & 1.00 & & & & & & \\
\hline Relative value arbitrage & 0.66 & 0.33 & 1.00 & & & & & \\
\hline Event driven & 0.82 & 0.51 & 0.74 & 1.00 & & & & \\
\hline Convertible arbitrage & 0.56 & 0.23 & 0.78 & 0.63 & 1.00 & & & \\
\hline Distressed securities & 0.68 & 0.42 & 0.77 & 0.84 & 0.63 & 1.00 & & \\
\hline Equity market neutral & 0.44 & 0.31 & 0.36 & 0.34 & 0.25 & 0.33 & 1.00 & \\
\hline Mergers arbitrage & 0.58 & 0.32 & 0.54 & 0.75 & 0.46 & 0.56 & 0.30 & 1.00 \\
\hline
\end{tabular}


Table 2: Out-of-Sample Evaluation Criteria of Monthly Rebalanced Portfolios (Period 1)

\begin{tabular}{|c|c|c|c|c|c|c|c|}
\hline & Return & Risk & CSR & Turnover & CVaR90 & CVaR95 & CVaR99 \\
\hline \multicolumn{8}{|c|}{ Panel A: Conservative Portfolio } \\
\hline $\mathrm{SC}$ & $0.35[0.40]$ & $0.71[0.71]$ & $0.49[0.56]$ & $0.99[0.63]$ & $0.37[0.36]$ & $0.59[0.58]$ & $1.02[1.00]$ \\
\hline IF & & $.67[0.66]$ & $0.49[0.56]$ & $0.62[0.20]$ & $0.32[0.30]$ & $0.52[0.50]$ & \\
\hline & 52[ & {$[0$.} & $.74[0.84]$ & 43.7 & $0.21]$ & & $0.90]$ \\
\hline ORTH & & 1] & $.52]$ & & 38] & & 02] \\
\hline DCC & $0.60]$ & $0.88[0.79]$ & $0.73[0.82]$ & $43.37[42.39]$ & $.27]$ & $.51]$ & $0.99]$ \\
\hline FLEX & 0.46[ & $0.61[0.59]$ & $0.78[0.82]$ & 50.69[ & $.10]$ & $.28]$ & $0.64]$ \\
\hline & 37] & .70 [0.69] & $7[0.57]$ & 20.56 & $.31]$ & $.52]$ & $0.93]$ \\
\hline EWMA & $0.45[0.44]$ & $0.53[0.51]$ & $0.87[0.87]$ & $6.24[3.41]$ & $.02]$ & & $0.48]$ \\
\hline \multicolumn{8}{|c|}{ Panel B: Aggressive Portfolio with Target Return $15.5 \%$ p.a. } \\
\hline $\mathrm{SC}$ & & $1.49[1.50]$ & $0.51[0.58]$ & & & & 2.75] \\
\hline IF & & $44[146]$ & & & 35] & & 2.63] \\
\hline RSDC & $0.61[0.67]$ & $1.01[0.90]$ & $0.75[0.87]$ & $34.80[22.53]$ & $0.57[0.38]$ & .65] & 1.49] \\
\hline ORTH & $0.74[0.79]$ & & & & & & \\
\hline DCC & $0.61[0.67]$ & $1.04[0.93]$ & $0.73[0.84]$ & $36.77[22.68]$ & $0.62[0.43]$ & $0.95[0.72]$ & $1.57[1.57]$ \\
\hline FLEX & & $1.01[0.98]$ & $0.73[0.78]$ & $31.73[23.97]$ & $0.57[0.50]$ & $0.89[0.81]$ & 1.50 [1.50] \\
\hline BEKK & $0.76[0.89]$ & 1.35 [1.35] & $0.57[0.69]$ & $17.21[13.85]$ & $1.15[1.15]$ & 1.57 [1.57] & 2.38 [2.38] \\
\hline EWMA & $0.76[0.87]$ & $1.04[1.03]$ & $0.74[0.81]$ & $6.43[4.11]$ & $0.62[0.61]$ & $0.94[0.92]$ & 1.57 [1.57] \\
\hline
\end{tabular}

Notes: The Table reports evaluation criteria for the out-of-sample monthly rebalanced conservative and aggressive portfolios for the period January 2002 to August 2005. The evaluation criteria are the mean and median values of realized return (Return), portfolio standard deviation (Risk), Conditional Sharpe ratio (CSR), portfolio turnover (Turnover) and Conditional value at risk at the 90\% (CVaR90), 95\% (CVaR95) and 99\% (CVaR99) confidence levels. Medians are reported in parentheses. 
Table 3: Out-of-Sample Evaluation Criteria of Monthly Rebalanced Portfolios (Period 2)

\begin{tabular}{lccccccc}
\multicolumn{1}{c}{ Return } & Risk & CSR & Turnover & CVaR90 & CVaR95 & CVaR99 \\
Panel A: & Conservative Portfolio & & & & & \\
SC & $0.20[0.54]$ & $0.74[0.71]$ & $0.32[0.74]$ & $0.99[0.63]$ & $0.54[0.46]$ & $0.77[0.68]$ & $1.22[1.10]$ \\
IF & $0.16[0.55]$ & $0.72[0.69]$ & $0.28[0.74]$ & $0.62[0.20]$ & $0.53[0.45]$ & $0.77[0.68]$ & $1.20[1.08]$ \\
RSDC & $0.21[0.49]$ & $1.10[0.97]$ & $0.69[0.81]$ & $43.75[36.86]$ & $0.97[0.75]$ & $1.32[1.05]$ & $1.99[1.64]$ \\
ORTH & $0.34[0.62]$ & $0.97[0.75]$ & $0.58[0.70]$ & $7.85[5.95]$ & $0.95[0.59]$ & $1.25[0.83]$ & $1.84[1.29]$ \\
DCC & $0.18[0.51]$ & $1.22[1.04]$ & $0.64[0.62]$ & $43.37[42.39]$ & $1.12[0.84]$ & $1.50[1.20]$ & $2.24[1.83]$ \\
FLEX & $0.29[0.44]$ & $0.73[0.69]$ & $0.46[0.69]$ & $50.69[46.54]$ & $0.48[0.43]$ & $0.71[0.66]$ & $1.16[1.09]$ \\
BEKK & $0.33[0.52]$ & $0.78[0.74]$ & $0.53[0.70]$ & $20.56[18.41]$ & $0.58[0.56]$ & $0.82[0.80]$ & $1.29[1.26]$ \\
EWMA & $0.31[0.53]$ & $0.68[0.59]$ & $0.69[0.80]$ & $6.24[3.41]$ & $0.43[0.31]$ & $0.65[0.51]$ & $1.08[0.89]$ \\
Panel B: Aggressive Portfolio with Target Return $\mathbf{1 3 . 5 \% ~ p . a . ~}$ & & & \\
SC & $0.23[0.53]$ & $1.30[1.18]$ & $0.26[0.45]$ & $5.29[4.59]$ & $1.30[1.03]$ & $1.72[1.40]$ & $2.53[2.53]$ \\
IF & $0.20[0.50]$ & $1.26[1.13]$ & $0.25[0.43]$ & $6.40[4.65]$ & $1.22[0.95]$ & $1.62[1.31]$ & $2.41[2.41]$ \\
RSDC & $0.11[0.51]$ & $1.32[1.03]$ & $0.64[0.65]$ & $30.94[18.64]$ & $1.33[0.77]$ & $1.76[1.09]$ & $2.58[2.58]$ \\
ORTH & $0.31[0.50]$ & $1.82[1.31]$ & $0.38[0.25]$ & $18.81[14.47]$ & $2.23[1.48]$ & $2.80[1.92]$ & $3.93[3.93]$ \\
DCC & $0.14[0.54]$ & $1.42[1.10]$ & $0.63[0.59]$ & $33.33[19.09]$ & $1.46[0.85]$ & $1.91[1.22]$ & $2.79[2.79]$ \\
FLEX & $0.27[0.43]$ & $0.96[0.87]$ & $0.45[0.47]$ & $31.44[25.32]$ & $0.72[0.54]$ & $1.04[0.82]$ & $1.65[1.65]$ \\
BEKK & $0.36[0.55]$ & $1.31[1.14]$ & $0.42[0.41]$ & $17.75[14.49]$ & $1.30[1.04]$ & $1.71[1.40]$ & $2.53[2.53]$ \\
EWMA & $0.35[0.45]$ & $0.98[0.80]$ & $0.61[0.57]$ & $6.85[4.00]$ & $0.80[0.43]$ & $1.12[0.70]$ & $1.76[1.76]$ \\
\hline
\end{tabular}

Notes: The Table reports evaluation criteria for the out-of-sample monthly rebalanced conservative and aggressive portfolios for the period September 2005 to September 2009. The evaluation criteria are the mean and median values of realized return (Return), portfolio standard deviation (Risk), Conditional Sharpe ratio (CSR), portfolio turnover (Turnover) and Conditional value at risk at the 90\% (CVaR90), 95\% (CVaR95) and 99\% (CVaR99) confidence levels. Medians are reported in parentheses. 